constances ou conditions affectant la saveur, l'arôme, l'odeur, la capacité de barattage, la texture, la couleur, la conservation, et les. défauts résultant du manque de conditions nécessaires ou de la présence des facteurs défavorables. L'exposé de ces études ne pourra. être fait que dans un travail plus étendu que celui que nous venons de présenter.

\title{
RECHERCHES SUR L'IDENTIFICATION DE LA GRAISSE: DE COCO (COCOSE OU COCOLINE) DANS LE BEURRE
}

par

L. HOTON

$D^{r}$ ès Sciences

On n'accorde plus aujourd'hui aucune importance scientifique aux compositions du beurre indiquées par Duclaux et Winter. BLYTH.

Les travaux d'Amberger, Groosfeld, Heidusda nous apportentmaintenant des résultats plus précis et qui sont exprimés en acides. gras, ce qui est plus logique que leur expression en glycérides ; de plus, ces travaux nous ont montré que, à côté des éléments identifiés par les auteurs anciens (oléine, palmitine, stéarine, butyrine, eaproïne, caprine et capryline), il existe également dans les beurres des acides laurique, myristique et caprinique, et certains, tels lelaurique et le myristique, en proportion notable.

$J$ 'ai réuni dans le tableau suivant les compositions généralement. admises aujourd'hui du beurre, de la cocose et du saindoux.

\begin{tabular}{|c|c|c|c|}
\hline & Beurre & Cocose & Saindoux \\
\hline Acide butyrique .......... & 4,3 &..- & \\
\hline Acide caproïque . . . . . . . . . & 1,6 & 0,5 & \\
\hline Acide caprylique $\ldots \ldots \ldots \ldots$. & 1,2 & 9 & \\
\hline Acide caprinique $\ldots \ldots \ldots \ldots$ & 1,2 & 4,2 & \\
\hline Acide laurique $\ldots \ldots \ldots \ldots \ldots$ & 5 & 48 & \\
\hline Acide myristique . . . . . . . . . . & 16,4 & 17,4 & \\
\hline Acide palmitique . . . . . . . . & 14,8 & 7,1 & 32,2 \\
\hline Acide stéarique $\ldots \ldots \ldots \ldots \ldots$ & 3,4 & 2,9 & 7,8 \\
\hline Acide oléique $\ldots \ldots \ldots \ldots \ldots \ldots$ & 44,8 & 4,7 & 60 \\
\hline Acide linoléique ................ & - & 0,9 & \\
\hline
\end{tabular}

Si l'on peut admettre une certaine fixité des éléments constituant le cocose et le saindoux, le beurre, au contraire, est soumis à des variations de eomposition saisonnières parfois très importantes: qui doivent modifier profondément les proportions respectives du tableau précédent.

En ce qui concerne le seul acide butyrique, l'expérience nous a 
montré que cet élément varie, au cours d'une année, de plus du simple au double dans les beurres de la même exploitation.

Et, si nous nous contentons des indications qui nous sont données par des indices que je qualifierai d'indices globaux, nous constatons que, outre l'acide butyrique, d'autres perturbations profondes affectent les autres composants.

Nous voyons en effet que la solubilité dans l'alcool (CRISMer) de l'ensemble des glycérides varie de $45^{\circ}$ à $62^{\circ}$, que les acides gras fixes vont de 85 à $93 \%$, que la réfraction (Abbe-Zeiss) descend à 38,8 et peut remonter à 47 , et que les acides gras volatiles (LEEFMANBEAM) vont de 15 à 38 .

Que représentent ces valeurs? Elles ne nous apportent aucune indication qualitative quant à la composition intime du beurre, et, au point de vue quantitatif, elles ne peuvent que nous permettre de préjuger de la prédominance de certains des constituants du beurre.

Dire que la prédominance de ces éléments doit être compensée par une déficience d'autres éléments est un truisme qui n'apporte aucune lumière. La thèse doctorale de M. G. Van BenEden ( $\mathrm{D}^{\mathrm{r}} \mathrm{Ph}$.) (Journal de Pharmacie de Belgique) est venue nous montrer que les dérivés de l'acide oléique existant dans le beurre fournissent l'élément compensateur des variations des autres glycérides.

La Revue Le Lait (février 1936) sous les signatures de M. le Professeur VIVARio et celle de M. Van BENEDEN a publié un travail qui confirme pleinement ces résultats. Je me borne à citer iei leur importante conclusion : "On constate que les acides à poids moléculaires moyens (caprylique, caprique, laurique et myristique) varient nettement et constamment en sens inverse des acides saturés à poids moléculaires élevés (palmitique, stéarique), sans que toutefois leurs variations se compensent complètement. La somme de ces deux groupes d'acides varie en sens inverse de l'acide oléique. Il en est de même des acides volatils solubles (butyrique et caproïque). On constate done que l'acide oléique compense réellement les variations de la somme des trois autres groupes d'acides, sans compenser cependant les variations de chacun des groupes pris isolément."

Cette constatation ouvre maintenant la voie à des recherches systématiques ayant au moins une base solide.

$$
*^{*} *
$$

Durant une année entière M. Van BENEDEN et moi avons, tous les quinze jours, analysé trois beurres authentiques d'origine différente : un beurre danois et deux beurres du pays herbager belge, dit Le Plateau d'Herve.

Nous avons estimé qu'une étude, pour être sérieuse, devait com- 
porter le cycle entier des variations annuelles et naturelles de composition. Nous avons choisi le beurre danois parce que nous étions certains d'avance que ses variations, tout au moins celles résultant des méthodes usuelles, ne seraient guère appréciables ; les beurres de Herve, au contraire, allaient, eux, nous montrer leurs habituels écarts et composition. Pour fixer d'emblée celles-ci, je dirai qu'au eours de l'année, l'indice des acides volatils solubles du danois n'a varié que de 30 à 33 , alors que les mêmes indices des deux autres beurres sont allés de 22,55 à 37,4 .

Chaque échantillon de beurre, après avoir été soumis aux méthodes habituelles de l'expertise, a été additionné de $10 \%$ de coco. M. Van BenEDEN les a soumis à la technique qu'il a imaginée ; de mon côté, j'ai appliqué celle que j'ai décrite (juin 1930, Annales des Falsifications).

L'addition de cocose en proportion inférieure à $15 \%$ n'apporte guère de modifications dans les normes habituellement utilisées par l'expertise. Je fais ici une exception bien nette pour la détermination des points de fusion des acétates de phytostérine ou de cholestérine qui sont caractéristiques. Mais, si l'on n'envisage que les modifications apportées par $10 \%$ de cocose aux chiffres des acides volatils solubles ou insolubles, bases de l'expertise actuelle, on doit reconnaître que ces modifieations sont d'un ordre peu important, et que de plus, elles manquent de régularité. Pour traduire ma pensée par des chiffres, je dirai qu'il arrivera souvent que l'influence de ces $10 \%$ de cocose se fera sentir dans un eas par un abaissement d'acides volatils solubles de la grandeur de 4 unités et de 1 unité seulement dans un autre cas ; les acides volatils insolubles, eux, augmenteront dans un cas de 0,5 et dans un autre de 2,5 pour cette même quantité de cocose ajoutée.

Les chiffres d'acides volatils solubles élevés ne peuvent pas nous donner la certitude de la pureté d'un beurre ; je cite ici, à titre d'exemple, un eas de fasification de beurre caractérisé par l'expertise de la comptabilité du prévenu, et par la présence dans la masse du beurre de minuscules grains de cocose qui avaient échappé au malaxage; les échantillons au nombre de 5 titraient de 30 à 31 A. V. S., et l'augmentation des A. V. I. n'avait rien de caractéristique. Il eût été, dans ce cas, interdit de conclure sur les seules données analytiques, et non seulement de conclure, mais même de soupçonner la falsification (1).

$$
*^{*} *
$$

En matière d'expertise du beurre, il est des chiffres qui nous

(1) La détermination des points de fusion des acétates des stérols est venue confirmer les conclusions de l'analyse. 
donnent, les uns une certitude, les autres un soupçon. Si on étudie les tableaux de M. Van BENEDEN, on constate que l'application de sa technique relative à l'indice corrigé aboutit aux résultats suivants :

Les 75 beurres purs qu'il a analysés durant un an ont présenté tous, à l'exception de 7 échantillons, des indices corrigés inférieurs à 65 . Les mêmes beurres, additionnés de $10 \%$ de cocose, avaient des indices corrigés supérieurs à 75 .

Restaient comme douteux 7 échantillons lesquels ont fait l'objet de l'application de la technique dont il sera question plus loin, ce qui a permis de réduire leur nombre à trois (trois sur cent cinquantesix).

L'importance de l'indice corrigé, c'est qu'il n'est en rien influencé par les changements saisonniers de la composition des beurres. Alors que les A. V. S. diminuaient dans les beurres du pays de Herve en juillet, août, septembre, l'indice corrigé était à peine abaissé.

$$
*^{*} *
$$

Dans l'étude publiée en 1930 par la Revue Internationale des Falsifications (juin), j'avais posé le principe d'une méthode pour l'identification, à la cocose, méthode basée sur la détermination de l'indice de réfraction des acides volatils insolubles recueillis lors de l'essai Polenske.

J'ai poursuivi ces essais durant un an, aux côtés de M. Van BENEDEN, sur les trois beurres qui faisaient l'objet de ses études.

Les déterminations ont été faites à l'aide du butyroréfractomètre de Zeiss et à la température de $25^{\circ}$, pour éviter les phénomènes d'interférence. Contrairement aux indications des fabricants du réfractomètre qui recommandent de garnir les prismes de matière grasse, j'ai constaté qu'il suffit d'une parcelle de celle-ci ( 2 mill.) pour éclairer le champ visuel. Le prélèvement se fait à l'aide d'un tube effilé.

Les différences de valeur de réfraction des deux matières grasses sont de l'ordre suivant: le cocose donne 14, les beurres 17 à 23,2 .

Mais il est à remarquer que si on fractionne la distillation de la graine de cocose en 5 ou 6 parties, les premiers fractionnements ont des valeurs inférieures à 14 ; elles tombent à 11,5 pour remonter à $12,2,13,14,15$.

Si maintenant on additionne les beurres de $10 \%$ de cocose, les valeurs sont inférieures à celles des beurres purs et s'établissent entre 15,2 et 21,5 ( 78 échantillons). Nous constatons, à propos de ces déterminations, les mêmes incertitudes que celles des essais LEEFman-Beam et PoLenske nous donnent : certains beurres purs présentent des chiffres semblables à ceux des beurres falsifiés par $10 \%$ de cocose, et la réciproque est également vraie.

Ces faits sont exceptionnels si on le veut, mais ils existent, et 
laissent l'analyste perplexe. J'ai cherché à réduire au minimum ces incertitudes en combinant les résultats de plusieurs essais.

Prenons un beurre pur, dont l'indice des acides volatils solubles (A. V. S.) est de 30, celui des acides volatils insolubles de 2,5 (A. V. I.) et l'indice de réfraction de ceux-ci de 20 (R. A. V. I.). Si nous l'additionnons de $10 \%$ de cocose, ces chiffres deviendront théoriquement $28,3,5,17$.

Posons les équations suivantes :

$$
\text { pur } \frac{30 \times 20}{25}=24 \text {, " cocotté " } \frac{28 \times 17}{35}=13,6 .
$$

Quelle valeur donner à ces quotients ?

Caractérisent-ils l'un la pureté, l'autre l'absence de cocose ?

Les trois facteurs de l'équation, pris isolément, manquent de précision; suis-je en droit d'en déduire une certitude qui serait basée sur trois déterminations manquant de fixité ?

Non certes! Mais il est permis d'extraire des résultats obtenus des chiffres caractéristiques, soit de la pureté soit de la présence de cocose, et de réserver comme douteux les quotients qui se placent entre les deux valeurs :

\begin{tabular}{|c|c|c|}
\hline & $\begin{array}{l}\text { Quotients des } 78 \\
\text { beurres purs }\end{array}$ & $\begin{array}{l}\text { Quotients des } 78 \\
\text { beurres cocottés }\end{array}$ \\
\hline Danois & 15 à 30 & 6 à 16 \\
\hline Vandeveen ........... & 15 à 31 & 8 à 22 \\
\hline Tanssens........ . . & 15 à 50 & 6 à 18 \\
\hline
\end{tabular}

(Je ferai remarquer que le beurre cocotté (VANDEVEEN) qui a donné 22 I. A. V. I. avait une composition anormale.)

Prenons dans les deux séries, les chiffres qui peuvent être considérés comme caractéristiques de la présence ou de l'absence de cocose. Me basant sur les 156 observations, j'estime qu'on peut considérer un quotient égal ou supérieur à 20 comme indiquant l'absence de cocose, et comme caractéristique de la présence de cette graisse, je prends le chiffre inférieur ou égal à 15 .

Restent les beurres dont les chiffres vont de 15 à 20 pour lesquels aucune conclusion ferme ne peut être assise ; ils sont au nombre de 20 sur les 156 échantillons.

Il est évident que l'analyste poussera pour ceux-là ses recherches plus loin, et que la détermination du point de fusion des stérols lui permettra d'arrêter une conclusion ferme. Mais, sans aller jusqu'à cette détermination, on peut recourir aux résultats de la technique de M. Van Beneden et l'appliquer à ces cas douteux; la réciproque est également vraie, certains résultats douteux obtenus par la détermination de l'Indice corrigé peuvent être complétés par l'application de ma méthode. 


\begin{tabular}{l|ll|ll|ll|l} 
R. ..... & 43 & 43 & 439 & 43 & 41,5 & 42 & 42 \\
A. V. S. . & 24,86 & 24,2 & 23,17 & 22,55 & 25,3 & 25,3 & 27,5 \\
A. V.I. . & 1,7 & 1,5 & 1,5 & 1,2 & 2 & 1,6 & 1,9 \\
A. F. ... & 89,5 & 89,7 & 90 & 89,6 & 88,1 & 89,5 & 89,2 \\
R. A. V.I. & 21,2 & 20,5 & 23,2 & 22,2 & 18,5 & 21 & 20 \\
Q. (1) ... & 31 & 28 & 36 & 27 & 23 & 33 & 29
\end{tabular}

A. V.S. .. 22,8

\begin{tabular}{l|l} 
A. V.I. & 2,2 \\
R. A. V. I. & 21,5 \\
22
\end{tabular}

R. $\ldots \ldots \mid 43 \quad 44 \quad 43,9 \quad 42,1 \quad 43$

A. V.S. . 23,65

A. V.I. .. 1,8

A. F. $\ddot{\text { V. I. }}{ }_{21,5}^{90,3}$

Q........ 28

\begin{tabular}{c|cc|c}
25,3 & 22,88 & 24,4 & 22,55 \\
2 & 1,9 & 1,8 & 1,7
\end{tabular}

$\begin{array}{llll}90,8 & 89,6 & 89 & 89\end{array}$

\begin{tabular}{l|l|l|l}
20,5 & 22,8 & 19,2 & 21 \\
28 & 25 & 24 & 28
\end{tabular}

\begin{tabular}{r|rr|r}
22,7 & 21,78 & 22,5 & 22,5 \\
2,8 & 2,2 & 3,5 & 2,5 \\
17,5 & 19 & 18 & 17,2 \\
14 & 18 & 12 & 15
\end{tabular}

\begin{tabular}{c|c}
23,2 & 25,9 \\
2 & 2,9 \\
18,8 & 18 \\
21 & 13
\end{tabular}

\begin{tabular}{l|ll|l|}
25,3 & 26,1 & 41 & 41,7 \\
\hline 1,9 & 1,7 & 29,7 & 30,8 \\
\hline & 1,2 & 2,5
\end{tabular}

\begin{tabular}{r|rr|r}
1,9 & 1,7 & 1,2 & 2,5 \\
88,8 & 89,5 & 87,7 & 86,3
\end{tabular}

\begin{tabular}{l|ll|l}
21 & 22 & 20 & 19 \\
22 & 33 & 50 & 30
\end{tabular}

\begin{tabular}{l|l}
42,2 & 41,6 \\
29 & 28,6 \\
1,3 & 2,6 \\
87,2 & 86 \\
20 & 19,5 \\
16 & 39
\end{tabular}

41,5

\begin{tabular}{l|l|l|l}
30,5 & 32,78 & 32,8 & 33,2
\end{tabular}

\begin{tabular}{rrr|r}
1,5 & 1,9 & 2,2 & 3,1
\end{tabular}

\begin{tabular}{lll|l}
87,8 & 88,1 & 86,4 & 88 \\
19,8 & 19,8 & 21 & 19
\end{tabular}

$15,\left.8\right|_{33} ^{19,8},$\begin{tabular}{ll|l}
21 & 19 & 20
\end{tabular} \begin{tabular}{l|ll|l|}
41 & $\left|\begin{array}{ll}42 & 41,7 \\
31,35 & 33,8\end{array}\right| 33$
\end{tabular} $\begin{array}{llll}2,2 & 2,6 & 3 & 2,8\end{array}$ \begin{tabular}{r|rr|r}
87,6 & 87,3 & 86,2 & 86,2
\end{tabular} $\begin{array}{lllll}19 & 18,5 & 17 & 18,5\end{array}$ \begin{tabular}{l|ll|l}
27 & 21 & 23 & 21,5
\end{tabular}

Mêmes beurres additionnés de $10 \%$ de coco

\begin{tabular}{ll|l|lll|l|l|l}
42,4 & 43,8 & 42,8 & 43 & 41,7 & 42,5 & 42,5 & 42,2
\end{tabular} \begin{tabular}{lll|ll|ll|l}
30,47 & 28,40 & 29,7 & 27,3 & 29,7 & 28 & 27,5 & 27
\end{tabular} \begin{tabular}{lllllll|l}
2,3 & 2,5 & 2,5 & 2,5 & 2 & 2,6 & 3 & 3,5
\end{tabular} \begin{tabular}{lllll|ll|l}
8,7 & 89,9 & 88 & 89,1 & 88,9 & 88,7 & 88,4 & 89,3
\end{tabular} \begin{tabular}{l|ll|ll|ll|l}
19,2 & 19,5 & 20 & 21 & 18 & 19 & 21,5 & 19,7 \\
26 & 22 & 30 & 23 & 22,5 & 20,5 & 19 & 15
\end{tabular}

A. V.I.

A. A. V. 18

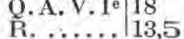

$23,6 \mid 21$

\begin{tabular}{r|r}
23,1 & 21,45 \\
3,8 & 4
\end{tabular}

$23,1 \quad 23,9$

3.128

\begin{tabular}{r|rr|r}
2,4 & 3,2 & 3,8 & 4 \\
17,8 & 20 & 17,7
\end{tabular}

\begin{tabular}{rrr|r}
3,4 & 3 & 2,5 & 3,7 \\
18 & 20 & 1,5 & 16,5
\end{tabular}

\begin{tabular}{r|r}
2,5 & 3,7 \\
17,5 & 16,5 \\
18 & 6
\end{tabular}

\begin{tabular}{r|cc|c}
29,2 & 27,5 & 31,38 & 33 \\
3,8 & 4 & 4,8 & 4
\end{tabular}

\begin{tabular}{r|r}
4,8 & 4 \\
16,2 & 16
\end{tabular}

13 12

\begin{tabular}{l|l}
12 & 13,5
\end{tabular}

\begin{tabular}{c|cc|cc|c}
34,65 & 32,5 & 31,9 & 29,15 & 26,4 & 27,5 \\
4 & 4 & 4 & 3,5 & 3,3 & 4,5
\end{tabular}

\begin{tabular}{c|cc|r}
4 & 4,5 & 4 & 3,5 \\
15,2 & 17,5 & 16 & 16,7 \\
13 & 15 & 10 & 13,5
\end{tabular}

\begin{tabular}{r|r}
$3,3,5$ & 17,5
\end{tabular}

\begin{tabular}{l|l}
16,5 & 17,5 \\
13
\end{tabular}

\section{Beurre danois}

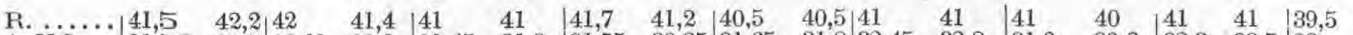

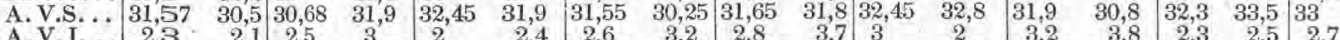

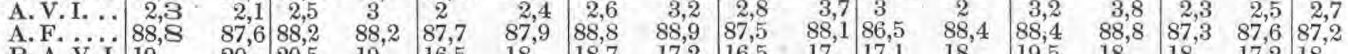

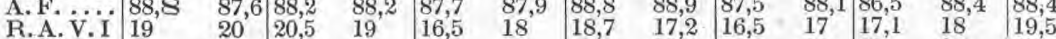

Q......... 26

\begin{tabular}{lllll|ll|l}
28 & 24 & 21 & 26 & 20 & 18,7 & 17,2 & 16,5 \\
23 & 16 & 50
\end{tabular}

\begin{tabular}{l|ll|l}
18 & 18 & 17,2 & 18 \\
30 & 25 & 23 & 22
\end{tabular} \begin{tabular}{l|ll|ll|ll|l}
40 & 41 & 40,9 & 41,5 & 40,3 & 42 & 41 & 43 \\
31,8 & 31,46 & 321 & 30,8 & 32,5 & 30 & 30,8 & 28,9
\end{tabular} \begin{tabular}{rrr|rr|rc|c}
2,1 & 2,4 & 2,4 & 3 & 2,2 & 3,3 & 3 & 2 \\
87,2 & 88,4 & 88,3 & 88,3 & 87,4 & 86,8 & 87,2 & 89,5
\end{tabular} \begin{tabular}{lll|lllll|l}
17,2 & 88,4 & 88,3 & 88,3 & 87,4 & 86,8 & 87,2 & 89,5 \\
18 & 19,5 & 21 & 18 & 18 & 195 & 20
\end{tabular} \begin{tabular}{lll|lllll}
30 & 24 & 30 & 22 & 26 & 17 & 20 & 28
\end{tabular}

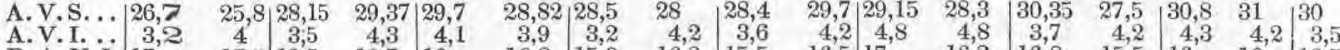

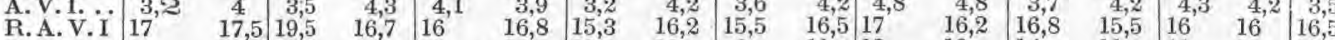

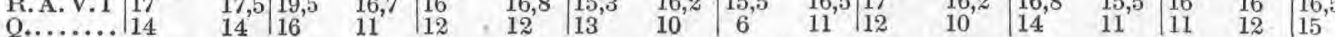

$28,6 \mid 29,1$

$4,3 \quad 4$

$10 \quad 12$
$27,5 \quad 28,5 \quad 29,15 \quad 27,7 \quad 27,2 \mid 27$ \begin{tabular}{c|cc|cc|c}
3 & 3,5 & 4,1 & 4,2 & 4,5 & 2,9 \\
16,5 & 17 & 15,5 & 16 & 17 & 17,1
\end{tabular} \begin{tabular}{l|ll|ll|l}
16,5 & 17 & 15,5 & 16 & 17 & 17,1 \\
15 & 14 & 11 & 10 & 10 & 15
\end{tabular}

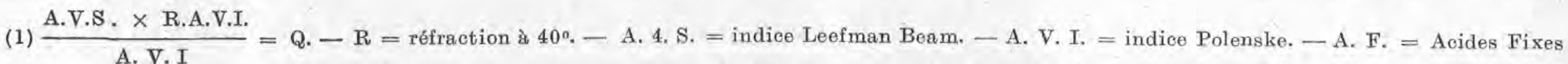
(Henher). - R. A. V. I. = réfraction à $25^{\circ}$ des acides volatils insolubles. 
Les deux techniques qui ont le mérite de fournir des résultats indépendants les uns des autres, arrivent à se compléter heureusement, puisque trois échantillons seulement, sur un total de 156, laissent un certain doute sur leur composition.

$$
*^{*} *
$$

L'examen du tableau montre que le beurre danois présente une assez grande régularité de composition, alors que les beurres de Herve provenant d'étables qui possèdent 30 à 32 vaches, subissent à partir du mois d'août les profondes modifications habituelles propres aux beurres de cette région.

Les déterminations R. A. V. I. ne sont en rien influencées par la diminution des A. V. S.

Les indices de réfraction, le taux des acides fixes descendent d'une manière générale quand les acides volatils montent, mais on observe cependant de fortes exceptions à ce qu'on a cru devoir ériger à l'état de dogme.

On remarquera également que la diminution des A. V. S. et l'augmentation des A. V. I. qui devrait logiquement résulter de l'addition de $10 \%$ de cocose, manque de régularité :

Exemple :

\begin{tabular}{|c|c|c|}
\hline \multirow[t]{2}{*}{ Beurres purs $\ldots \ldots \ldots \ldots \ldots \ldots \ldots \ldots$} & 32,7 & 32,8 \\
\hline & 1,9 & 2,2 \\
\hline Beurres cocottés ................. & 29,2 & 30 \\
\hline & 4,4 & 3,5 \\
\hline
\end{tabular}

Les quotients varient, pour les mêmes beurres, de quinzaine en quinzaine, sans qu'on puisse trouver une explication à ces changements.

Il apparaît, en tous cas, que l'indice de réfraction des acides volatils insolubles constitue une valeur indépendante, et c'est à ce titre qu'elle doit être envisagée.

De plus e'est cet indice qui se présente comme ayant le plus de fixité.

$\mathrm{Si}$, en effet, je rapporte à 100 les différences entre les variations maximum et minimum des trois indices A. V. S., A. V. I., R. A. V. I. c'est ce dernier qui présente le plus de fixité.

$$
*^{*} *
$$

Le vieil axiome de l'interdépendance des différents indices a eneore quelques partisans. Je l'ai combattu il y a plus de 30 ans déjà ; les constatations de M. Van BENEDEN sur l'importance compensatrice des dérivés de l'acide oléique apportent une preuve de plus à ma thèse. 
Si on consulte les graphiques publiés annuellement par la Station de contrôle du beurre de la Frise (Hollande), on est frappé par ce fait : alors que d'avril à novembre les A. V. S. marchent en sens inverse de la réfraction et cela non pas d'une manière absolue, mais cependant dans une relation assez étroite, au contraire, à partir de novembre jusqu'en mars, les A. V. S. remontent et la réfraction reste quasi invariable.

Je constate que pour la période 1934-35, de novembre à mars, les A. V. S. montent de 26,5 à 32,5 , et que la réfraction ne varie que de 42,4 et 42,2 . Ces différences n'auraient qu'une valeur relative s'il s'agissait de beurre provenant d'une exploitation moyenne, mais elles acquièrent une importance considérable si on les rapporte à l'énorme quantité de 22.000.000 de kilogrammes auxquels elles s'appliquent.

Et ce manque de concordance se remarque tous les ans à la même époque en Frise.

Certains auteurs affirment encore aujourd'hui l'influence de l'alimentation par les tourteaux de cocose sur la composition du beurre. J'ai entendu affirmer par un de nos experts les plus réputés que "puisque les tourteaux de cocose renfermaient de la laurine, le lait devait fatalement renfermer de l'acide laurique ". Cette affirmation produite, lors d'un procès célèbre, n'était il est vrai appuyée d'aucune preuve.

J'ai procédé à des expériences comparatives dans deux étables d'une quinzaine de vaches chacune; dans l'une d'elles, les vaches recevaient tous les jours, de $1 \mathrm{~kg}$ et demi à $2 \mathrm{~kg}$. de cocottier. Les différents beurres n'ont montré, au cours des expériences qui ont eu une durée d'un mois, aucun caractère spécifique du coco, les quantités d'A. G. V. I. n'étaient en rien augmentées là où le coco était utilisé.

$$
*^{*} *
$$

Quelle conclusion pratique faut-il tirer de mes recherches?

Loin de moi la prétention de présenter ma méthode comme infaillible pour déceler la cocose dans un beurre quelconque. En dehors de la détermination des stérols, il n'existe aucune méthode certaine permettant de déceler moins de $15 \%$ de cette graisse ; mais mon procédé, comme celui de M. Van BENEDEN, permet d'éliminer d'emblée, et dans des cas très nombreux, l'hypothèse d'une falsification par la cocose, graisse qui est particulièrement difficile à identifier quand elle se trouve à faible dose, tant à cause de ses caractères chimiques, qu'à cause du manque de précision des techniques de l'analyse.

Et, c'est à ce titre que, dans l'étude de nos travaux, je présente mes recherches au jugement de mes confrères. 